\title{
DIE NEUE AKTIVITATT DER BLOCKFREIEN LÄNDER AUF DER INTERNATIONALEN BÜHNE
}

\author{
Von Ranko PetKovic
}

I.

Vom 8. bis 12. Juli 1969 fand in Belgrad ein Beratungstreffen der blockfreien Länder statt. Es stellt nach einer Zeitspanne der Passivität und sogar einer gewissen ideen- und organisationsmäßigen Desorientation einen der bedeutenden Wendepunkte beim Aktivieren dieser Länder auf der internationalen Bühne dar ${ }^{1}$. Gewisse Länder sahen in der Nichtzugehörigkeit zu den militärisch-politischen Bündnissen der Großmächte eine Chance, in der Nachkriegswelt bei der Festlegung der Wege ihrer inneren Entwicklung und ihrer Außenpolitik unabhängig zu bleiben; und vice versa: sie stellten die Einordnung in Blöcke einer Unterordnung unter die Hegemonie der in Washington und Moskau formierten militärischen, wirtschaftlichen und politischen Zentren der Macht gleich.

Dieser Standpunkt hätte gewissermaßen die Reaffirmation der Doktrin der Neutralität sein können. Obwohl viele Ansatzpunkte juristischer und politischer Natur für die Herstellung solcher Parallelen bestehen, ist die Blockfreiheit keine gewöhnliche Reaffirmation der klassischen Neutralität, und zwar, weil die Politik der Blockfreiheit im Unterschied zur Neutralitätspolitik ein Plädoyer für Aktionsfreiheit, Aktivität, Engagiertheit in den internationalen Beziehungen darstellt. Wenn Passivitä t und Äquidistanz in der Vergangenheit eine Methode zur Realisierung der lebenswichtigen nationalen Interessen neutraler Länder sein konnten, so sahen die blockfreien Länder den einzigen Weg zur Wahrung ihrer Unabhängigkeit, den einzigen Ausweg aus den Spannungen des Kalten Krieges im a k t i ve n Wirken gegen die Blockpolitik und gegen die Politik der Stärke überhaupt ${ }^{2}$.

Diese Politik würde sich auf isolierte Oasen beschränken ohne weitreichendere Wirkung auf die internationalen Beziehungen, wenn nicht parallel mit der Bipolarisation der Welt der Destruktionsprozeß des Kolonialsystems verlaufen wäre, der dazu geführt hat, daß auf der internationalen Bühne ein dritter Faktor in Erscheinung tritt, die neubefreiten Länder Asiens und Afrikas ${ }^{3}$. Diese Länder haben sich fast alle für die Politik der Nichteinordnung in die militärisch-politischen Blöcke der Großmächte entschieden ${ }^{4}$. Die ehemaligen Kolonien wollten nicht eine Form der Unterordnung durch eine andere Form der Unterordnung ersetzen. Von ihrem Standpunkt aus wäre der Beitritt zu militärisch-politischen Bündnissen der Großmächte dasselbe gewesen wie der Verlust der Unabhängigkeit, die sie soeben erst errungen hatten. Das könnte ihre nationalen Interessen gefährden.

Weil die Entkolonisierung zuerst in Asien begonnen hatte, war die Blockfreiheit in der ersten Zeit ein asiatisches Phänomen: daher in der ursprünglichen Version eine gewisse Nehrusche Note der Friedensstiftung und Vermittlungsbereitschaft.

1 Vgl. Philippe Herrman im Pariser „Le Monde“ vom 9. Juli 1969 und Giovanni Lovizetti im Mailänder "Relazioni internazionali“ vom 19. "Juli 1969.

2 Khalid I. Babaa: „Non-alignment and Neutralism“, Kuzzent Events, March 1969, Bombay.

3 Bimla Prasad: „The General Experience of Nonalignment and its Prospects for the Future“, Nonalignment in the World of Today, Belgrad 1969.

4 Bojana Tadić: "Nonalignment - a Conceptual and Historical Survey“, Nonalignment in the World of Today, Belgrad 1969. 
Zusammen mit der Übertragung des Epizentrums auf Afrika und der Erscheinung einer großen Zahl neuer unabhängiger afrikanischer Staaten erhielt die Blockfreiheit bestimmte afrikanische Akzente, die in einer größeren Dosis an Radikalismus und Dynamik zum Ausdruck kamen.

Die Politik der Blockfreiheit als Politik der Unabhängigkeit kann aber nicht ausschließlich auf die bisher erwähnten Prozesse oder Regionen beschränkt bleiben. Sie läßt sich weder politisch noch geographisch lokalisieren. Sie kann sich politisch nicht ausschließlich auf den Entkolonisierungsprozeß beschränken, ebensowenig wie geographisch auf den asiatischen oder afrikanischen Boden. Denn dasselbe Bestreben, eine vollständige nationale Unabhängigkeit zu erzielen und in den internationalen Beziehungen aktiv zu wirken, tritt auch an anderen Orten und unter anderen politischen Verhältnissen zutage. Von diesem Bestreben ist beispielsweise die unvermeidbare historische Schwächung des USA-Einflusses in Lateinamerika begleitet, wo von einzelnen politischen Bewegungen oder Regierungen die Prinzipien und Ziele akzeptiert werden, die weitgehend mit denjenigen übereinstimmen, die vom Begriff der Blockfreiheit erfaßt sind.

Selbst wenn bestimmte internationale Umstände nicht zu einer unerläßlichen synchronisierten internationalen Aktivität der blockfreien Länder beigetragen hätten, würde die Schaffung verschiedener Formen der Zusammenarbeit zwischen ihnen einen selbstverständlichen Schritt darstellen. Ein ähnliches historisches Schicksal der asiatischen und afrikanischen Länder, ähnliche Verhältnisse, die in diesen Ländern unmittelbar nach Erlangung der Unabhängigkeit geherrscht haben, ähnliche Ziele, denen nicht nur diese, sondern auch viele andere, den militärpolitischen Allianzen der Großmächte ferngebliebene Länder, zustreben - all das bildet die objektive Grundlage für ihre gegenseitige Annäherung und Verständigung. Diese ganz natïrliche Voraussetzung zur Herstellung und Erweiterung ihrer gegenseitigen Beziehungen stellte jedoch nur den Ausgangspunkt dar, um bestimmte Formen für die Einleitung gemeinsamer Aktionen auf der internationalen Bühne zu schaffen. Bedeutsam sind aber auch $u$. a. die folgenden Ursachen gewesen:

\section{Die Aggressionsgefahr}

Die Gefahr eines neuen Weltkrieges und neuer lokaler kriegerischer Auseinandersetzungen, die einem neuen Weltkrieg den Weg bahnen, gefährdet, zum erstenmal in der Geschichte, nicht nur einzelne Länder, Regionen oder Kontinente, sondern beginnt durch die Erfindung von Atom- und anderen Massenvernichtungswaffen, das ganze Menschengeschlecht zu bedrohen.

\section{Die Politik der Stärke}

Die neubefreiten Länder und auch andere, die sich für die Politik der Unabhängigkeit entschieden haben, sind zur Zielscheibe eines starken Drucks der Großmächte geworden. Die Großmächte waren bemüht, diese Länder unter ihren Einfluß zu bringen. Außerdem hatten die Kolonialmächte auf alle mögliche Art und Weise versucht, den Entkolonisierungsprozeß zu verhindern bzw. aufzuhalten, oder aber den früheren Herrschafts- und Ausbeutungsverhältnissen eine neue Form zu geben. Im Kontext des Kalten Krieges hatte die Politik der Stärke eine ständig wachsende 
Bedeutung erlangt und die unabhängigen und fortschrittlichen Bewegungen in den Kampf um das nackte Leben getrieben sowie gebieterisch vor die Notwendigkeit gestellt, verschiedenen Formen des politischen, wirtschaftlichen und militärischen Drucks entgegenzutreten.

\section{Die Verschärfung des wirtschaftlichen Mißverhältnisses}

Die historische Entwicklung hat zu krassen Unterschieden in der Entwicklungsstufe einzelner Länder und ganzer Kontinente geführt. Anstatt im Rahmen der gegenwärtigen internationalen Gemeinschaft, die über die unerläßlichen wirtschaftlichen Möglichkeiten und technischen Mittel verfügt, diese Unterschiede zu lindern und allmählich aus der Welt zu schaffen, schlug die Entwicklung eine entgegengesetzte Richtung ein, so daß die zwischen den entwickelten und unterentwickelten Ländern bzw. Kontinenten bestehende Kluft immer tiefer wird. Am schwersten betroffen durch derartige Zustände sind freilich die neubefreiten Länder. Sie haben zwar die für eine raschere wirtschaftliche Entwicklung unbedingt erforderliche Hilfe erhalten, sind aber dafür zum Gegenstand des neokolonialistischen Drucks geworden, durch den sie zur Bindung an bestimmte Großmächte gezwungen werden sollen, unter gleichzeitiger Beschränkung ihrer Unabhängigkeit auf eine bloße Fiktion. Diese wirtschaftliche Kluft und eine solche Stellung der Entwicklungsländer haben dazu geführt, daß die Teilung der Welt in den reichen Norden und den armen Süden zu einer von jenen Demarkationslinien wird, die katastrophale Folgen zeitigen können.

II.

\section{Das Beratungstreffen von Belgrad}

Drei besondere Umstände führten zur Einberufung des Beratungstreffens der blockfreien Länder in Belgrad:

a) die Erkenntnis, daß ihre Versammlungen in der Vergangenheit, die Belgrader Konferenz 1961, die Wirtschaftskonferenz in Kairo 1962, die Kairoer Konferenz 1964 bedeutende Auswirkungen in den internationalen Beziehungen gehabt hatten ${ }^{5}$;

\footnotetext{
5 Wir wollen nur einige internationale Aktionen erwähnen, die direkt oder indire:- aus den Initiativen und Forderungen der blockfreien Länder hervorgegangen sind:

1. Unter der Schirmherrschaft der Vereinten Nationen erfolgte die Einleitung einer umfassenden Aktion zur Lösung des Problems der Weltwirtschaft und Verbesserung der Stellung der Entwicklungsländer. Dies führte zur Bildung der 77-Länder-Gruppe, zur Abhaltung zweier Weltwirtschaftskonferenzen und zur Schaffung entsprechender ständiger Mechanismen, die in Übereinstimmung mit diesen Zielen wirken sollen. 2. Ergreifung von Initiativen und Einleitung von Aktionen auf dem Gebiet der Abrüstung. Dies hatte die Bildung einiger, auch die blockfreien Länder mit einschließenden, Organisationen sowie das Zustandekommen bestimmter Vereinbarungen und Verträge im Bereich der nuklearen Abrüstung und friedlichen Nutzung des Weltraums zur Folge.

3. Einleitung eines politischen und verfassungsmäßigen Verfahrens zur Anpassung der UNO-Mechanismen an die neuen internationalen Verhältnisse. Dieser Schritt zeitigte folgende Ergebnisse: Der Sicherheitsrat und der Wirtschafts- und Sozialrat wurden durch Vertreter der neubefreiten Länder Asiens und Afrikas erweitert; an verschiedenen Einrichtungen und Organisationen der UNO sind bestimmte strukturelle und andere fortschrittliche Änderungen vorgenommen worden.

4. Energische Aktion zur vollständigen Durchführung der Entkolonisierung und zur Bekämpfung der Rassendiskrimination. Das Ergebnis: territoriale Verkleinerung des Gebiets, in dem Kolonialismus und Rassentrennung herrschen, bzw. Einengung desselben auf die letzten größeren Stützpunkte im mittleren und im südlichen Teil des afrikanischen Kontinents.

5. Einleitung einer Aktion zur Kodifizierung der Prinzipien der aktiven und friedlichen Koexistenz, was zu folgenden Resultaten führte: Bildung bestimmter Komitees in den Vereinten Nationen; Abhaltung einer ganzen Reihe von Konferenzen, auf denen ein gewisser Fortschritt bei der Formulierung der von diesem Begriff erfaßten Rechtsnormen erzielt werden konnte.
} 
b) die Erkenntnis, daß in den letzten Jahren die Passivität der blockfreien Länder und das Fehlen gemeinsamer Aktionen zur Verschlechterung ihrer Stellung in den internationalen Beziehungen beigetragen haben und

c) das immer skrupellosere Verhalten der Großmächte in der Weltpolitik, das in bewaffneten Interventionen, Einmischung in innere Angelegenheiten kleiner und mittlerer Länder in „ihren“ Einflußsphären usw. zum Ausdruck kam.

Nach umfangreichen diplomatischen Sondierungen, bei denen Jugoslawien besonders aktiv war, kam es zu dem Treffen in Belgrad. Schon allein die Zahl der Staaten (51), die ihre Delegationen entsandten ${ }^{6}$, zeigte, daß die Idee der Blockfreiheit keine überholte politische Kategorie ist, deren Schwanenlied, wie viele behaupteten, auf der Kairoer Konferenz 1964 gesungen worden sein soll. Is stellte sich heraus, daß in der fast fünfährigen Zeitspanne der mehr oder weniger großen Passivität der blockfreien Länder kein einziges von ihnen und auch nicht von jenen, die inzwischen die Unabhängigkeit erlangt hatten, sich für die Politik der Einordnung in die Blöcke entschieden hatte.

Eine Analyse der Zusammensetzung der Teilnehmerländer zeigt folgendes:

a) daß vier Kontinente vertreten waren - Afrika (33), Asien (8), Lateinamerika

(3) und Europa (2);

b) unterschiedliche Formen der Staatsordnung - Monarchien (8), Republiken (43) und unterschiedliche Gesellschaftssysteme - kapitalistische und sozialistische sowie jene, von denen man weder sagen kann, ob sie kapitalistisch noch ob sie sozialistisch sind;

c) daß sich Länder versammelten, deren Beziehungen zu den Großmächten sehr gut sind und solche, die sehr schlechte Beziehungen zu den Großmächten haben, und zwar sowohl im Hinblick auf die USA als auch im Hinblick auf die Sowjetunion oder China;

d) daß sich Länder versammelten, die in bezug auf ihre geographischen und demographischen Merkmale sehr verschiedenartig sind - von Indien (530 Millionen Einwohner) bis Kuwait (500000), die aber alle sogenannte Entwicklungsländer sind;

e) daß sich einige von ihnen im Kriegszustand befinden (arabische Länder) und in einigen der Bürgerkrieg tobt (Nigeria);

f) daß sich verschiedene Religionen, Ideologien und Zivilisationen begegnet sind.

Unterschiede, formale und wesentliche, die aus der angeführten Klassifikation hervorgehen, können zu zwei widersprüchlichen Schlußfolgerungen veranlassen: erstens - daß diese Unterschiede zu der Uneinigkeit und Unwirksamkeit der blockfreien Länder führen oder zweitens - daß sie in Wirklichkeit die Breite, Flexibilität und Dogmenlosigkeit der Plattform der Blockfreiheit bestätigen. Die erste Schlußfolgerung kann nur bis zu einer bestimmten Grenze angenommen werden, weil Einheit und Wirksamkeit nicht unbedingt aus der Gleichheit der staatlichen und gesellschaftlichen Systeme und politischen Meinungen hervorgehen müssen. Die zweite Schlußfolgerung ist annehmbarer: die blockfreien Länder begründen ihre

6 Zum Beratungstreffen waren die Länder eingeladen, die sich 1964 an der Kairoer Konferenz beteiligt hatten (aus verschiedenen Gründen folgten Kuba, Dahomey, Finnland, Mexiko, Saudi-Arabien und Togo nicht der Einladung, ohne dadurch die Frage ihrer Beteiligung an der Gipfelkonferenz oder an anderen Aktivitäten der blockfreien Länder zu präjudizieren) und Länder, die inzwischen die Unabhängigkeit erlangt hatten.

Anwesend waren folgende Länder: Afghanistan, Algerien, Burma, Burundi, Kambodscha, Kamerun, Anwesend waren folgende Länder: Afghanistan, Algerien, Burma, Burundi, Kambodscha, Kamerun, Ghana, Guinea, Indien, Indonesien, Irak, Jamaika, Jordanien, Jugoslawien, Kenia, Kuwait, Laos, Liberia, Libanon, Libyen, Malawi, Mali, Marokko, Nepal, Nigeria, Uganda, VAR, Jemen, Mauretanien, Tansania, Senegal, Sierra Leone, Somaliland, Sudan, Syrien, Tschad, Tunesien, Zambia und in der Eigenschaft als Beobachter: Argentinien, Bolivien, Brasilien, Chile, Trinidad und Tobago, Uruguay und Venezuela. 
Aktionseinheit nicht auf den Prinzipien der blockmäßigen Versammlung, die von den gleichen ideologischen Postulaten inspiriert wird, sondern auf der Gleichheit der lebenswichtigen nationalen Interessen und Bestrebungen der Länder, die sich für den Frieden, die Unabhängigkeit und Entwicklung einsetzen.

Der Verlauf und die Beschlüsse des Beratungstreffens haben dies bestätigt. Obzwar die Sitzungen geschlossen waren, kann aufgrund glaubwürdiger Informationen angenommen werden, daß zwei Tendenzen in der Debatte deutlich geworden sind: Die erste Tendenz, besonders bei den Verfahrensfragen, zeigt eine ganze Skala unterschiedlicher Anschauungen; die zweite Tendenz zeugt davon, daß in wesentlichen Fragen die große Mehrheit ohne größere Schwierigkeiten eine gemeinsame Sprache finden kann? ${ }^{7}$ Sogar die Länder, die ziemlich scharf ihre Vorbehalte in bezug auf die politische Plattform der erneuten Versammlung und der Aktivierung der blockfreien Länder, besonders aber im Hinblick auf die Einberufung eines neuen Gipfeltreffens, geltend gemacht haben (einerseits Algerien und andererseits Senegal), unterzeichneten das Schlußkommuniqués, in dem es im wesentlichen heißt:

- die Hingabe aller Teilnehmerländer an die Politik der Blockfreiheit wurde bestätigt. Es wurde betont, daß die Prinzipien der Blockfreiheit, die 1961 in Belgrad und 1964 in Kairo proklamiert worden sind, auch unter den jetzigen Bedingungen ihren vollen Wert behalten;

- die Politik der Stärke in allen Formen wurde verurteilt, besonders aber Gewaltaktionen des Imperialismus, Kolonialismus und Neokolonialismus;

- dem Befreiungskampf der Völker Vietnams, der portugiesischen Besitzungen, Palästinas, Südafrikas und Rhodesiens, die um Freiheit und um das Recht auf Selbstbestimmung kämpfen, wurde Utnerstützung gewährt, und es wurde das Einverständnis mit der Resolution des Sicherheitsrats vom 22. November 1967 zum Ausdruck gebracht, worin die Zurückziehung Israels aus den okkupierten Territorien der arabischen Länder verlangt wird;

- es wurde die Notwendigkeit hervorgehoben, dringende Maßnahmen zwecks Milderung der Disproportionen in der Weltwirtschaft zu unternehmen, besonders im Rahmen der Vorbereitungen für die Zweite Entwicklungsdekade, der Tätigkeit der UNCTAD- und der 77-Länder-Gruppe;

- die Ergebenheit gegenüber den Prinzipien der UNO-Charta wurde bestätigt, und eine. größere Wirksamkeit der UNO sowie die Rückgabe der legitimen Rechte an die VR China verlangt;

- es wurde die Auffassung vertreten, daß die gegenwärtige internationale Situation eine größere Aktivität der blockfreien Länder und aller Kräfte erfordert, die sich für die Unabhängigkeit, Souveränität, Nichteinmischung, Abschaffung des Kolonialismus und der Rassendiskriminierung einsetzen, sowie für die Beschleunigung der Wirtschaftsentwicklung und für die Gleichberechtigung in den internationalen Beziehungen;

- es wurde der Wunsch ausgesprochen, daß alle Länder, die ihre Zugehörigkeit zur Politik der Blockfreiheit proklamiert haben, vor allem jene, die nach der Kairoer Konferenz die Unabhängigkeit erlangt haben, sowie alle Mitglieder der Organisation für Afrikanische Einheit zum Treffen der blockfreien Länder im Einklang mit den Prinzipien und Kriterien eingeladen werden, die auf der Belgrader und der Kairoer Konferenz angenommen worden sind.

7 Miroslav Kreačić: „Die Aktionsrichtungen“, „Internationale Politik“, 5.-20. August 1969, Belgrad.

8 Wortlaut des Kommuniqués in der ,Internationalen Politik“, 5.-20. August 1969, Belgrad. 
Das Kommuniqué ist in Wirklichkeit eine Synthese der in den vier Vorentwürfen enthaltenen Gedanken, die von den Delegationen Zambias, Kenias, Algeriens und Jugoslawiens eingebracht wurden sowie der Standpunkte, die in den Schlußdebatten im Redaktionskomitee vorgetragen worden sind.

Die jugoslawische Diplomatie war besonders beim Suchen nach Kompromissen aktiv. Den größten Beitrag zum erfolgreichen Ausgang des Treffens gaben aber, nach jugoslawischen Bewertungen, die Länder des schwarzen Afrika: in Situationen, da die Debatten über Verfahrensfragen das Treffen von den wesentlichen Problemen abzulenken drohten, traten die afrikanischen Vertreter solidarisch mit der These auf, daß der Zweck des Treffens nicht darin liege, Unterschiede, die in den Auffassungen der Teilnehmerländer bestehen, zum Ausdruck zu bringen oder zu potenzieren, sondern die Ansatzpunkte für eine gemeinsame Aktion zu fixieren?.

Die politischen Wirkungen des Beratungstreffens werden schon aus folgenden Punkten deutlich:

1. Das Treffen zeigte, daß die blockfreien Länder eine zahlenstarke Gruppierung darstellen, deren Zahl sich trotz der Schwierigkeiten, denen sie sich in ihrer inneren Entwicklung und in den internationalen Beziehungen gegenübersehen, nicht verringert, sondern konstant vergrößert.

2. Das Treffen hat den einmütigen Willen der blockfreien Länder bestätigt, ihre Aktivität in den internationalen Beziehungen im Kampf für Frieden, Unabhängigkeit und Entwicklung zu koordinieren und zu verstärken.

3. Das Treffen formulierte die Hauptelemente der Plattform und die Grundrichtungen der Aktionen der blockfreien Länder im kommenden Jahrzehnt.

III.

Der Zweck des Beratungstreffens lag vor allem darin, die Idee multilateraler Tretfen auf verschiedenen Ebenen zu fördern und möglichst vielgestaltige Formen der Aktivität der blockfreien Länder in der Zukunft anzuregen.

Eine erste Folge war das Ministertreffen der blockfreien Länder vom 27. September 1969 in New York, das im Rahmen der XXIV. Tagung der UNO-Generalversammlung stattfand. Dem Ministertreffen wohnten die Vertreter von 59 Ländern bei. Das ist die größte Zahl von Ländern, die bisher auf einem Treffen der Blockfreien vertreten waren ${ }^{10}$. Zweifelsohne hat dazu in großem Maße der Umstand beigetragen, daß die Vertreter dieser Länder sich in New York zur Tagung der Generalversammlung eingefunden hatten, was aber nicht im mindesten die Bedeutung ihres Beschlusses schmälert, sich separat zu treffen und in einer Arbeitsatmosphäre Beschlüsse von weitreichender Bedeutung für die Fortsetzung und Konkretisierung der in Belgrad in Gang gebrachten Initiativen zu fassen.

Das Treffen in New York, das nur etwa vier Stunden dauerte, bestätigte evidenter als je ein Treffen der blockfreien Länder bisher, daß Unterschiede in den Meinungen rasch in den Hintergrund geraten, wenn unmittelbar über Fragen von lebenswichtiger Bedeutung beschlossen wird. Ohne Zeitaufwand für Randfragen

\footnotetext{
9 Auszüge aus den Reden aller Diskussionsteilnehmer in der „Internationalen Politik“, 5.-20. August 1969, Belgrad.

10 Es beteiligten sich alle Länder, die dem Beratungstreffen in Belgrad beigewohnt haben, außer Burma, Senegal und Uruguay sowie 11 Länder, die nidłt auf dem Belgrader Treffen vertreten waren: Guayana, Peru, Südjemen, Singapur, Malaysia, Dahomey, Äquatorial-Guinea, Gambia, Togo, Saudi-Arabien.
} 
faßten die Minister Beschlüsse, die auch die größten Skeptiker zwingen werden, ihre Meinungen über den eng begrenzten Raum, der für die gemeinsame Aktion der blockfreien Länder besteht, zu revidieren:

1. Sie bestätigten die wesentlichen Elemente der politischen Plattform, von der aus sich die gegenwärtige Aktivität der blockfreien Länder entwickelt;

2. Sie stimmten der Idee zu, daß der 25. Jahrestag der Vereinten Nationen für die Bekräftigung der Prinzipien und Ziele der UNO-Charta benützt wird, die in ihrem Wesenskern mit den Prinzipien und Zielen der Politik der Blockfreiheit identisch sind, und gründeten ein Unterkomitee, zusammengesetzt aus Vertretern Äthiopiens, Jugoslawiens, des Irak und Ghanas, das konkrete Vorschläge in diesem Sinne vorbereiten wird;

3. Sie erzielten Einvernehmen über die Abhaltung des Vorbereitungstreffens für die Gipfelkonferenz im Frühjahr 1970 in Dar es-Salam, auf der unter anderem darüber beschlossen wird, welche Länder zum Gipfeltreffen eingeladen werden ${ }^{11}$;

4. Sie kündigten an, daß die neue Gipfelkonferenz der blockfreien Länder 1970 wahrscheinlich in Neu-Delhi stattfinden soll.

In Anbetracht dessen, daß noch vor wenigen Monaten sogar darüber, ob Indien für oder gegen ein neues Gipfeltreffen ist, berechtigte Zweifel herrschten, und in Belgrad die Delegation Algeriens alles getan hat, um zu beweisen, daß die Abhaltung einer neuen Konferenz der Blockfreien inopportun wäre, zeugt der einmütige Beschluß des Ministertreffens über die Zweckmäßigkeit und die Termine der Treffen in Dar es-Salam und in Neu-Delhi von der immer höheren Stufe der Aktionseinheit der blockfreien Länder in der gegenwärtigen internationalen Konstellation.

11 In Anbetracht dessen, daß gewisse Länder, die zu militärpolitischen Bündnissen der Großmächte gehören, in ihrer politischen Praxis faktisch für die Prinzipien und Ziele plädieren, für die sich die blockfreien Länder einsetzen, warf sich auch die Frage ihrer eventuellen Einladung zur Gipfelkonferenz auf. Das betrifft besonders Pakistan, das den Wunsch aussprach, sich den Aktivitäten der blockfreien Länder anzuschließen. Damit wurde eine wichtige prinzipielle Frage in Gang gebracht: kann ein Land, das einem Block angehört, als blockfrei behandelt werden? Formal logisch, wäre die Antwort negativ. Vom Standpunkt der Breite und Wirksamkeit der politischen Aktion der Blockfreien könnte die Antwort positiv sein. Die Sache wird komplizierter, wenn in dieses prinzipielle oder theoretische Problem die Antagonismen Indiens und Pakistans eingeschaltet werden. In Anbetracht dessen nahm Pakistan von der Forderung Abstand, dem Ministertreffen in New York beizuwohnen, doch wird die Frage seiner Teilnahme sowie der Beteiligung anderer Länder am Gipfeltreffen in Dar es-Salam erörtert werden. 